\title{
REGULARITY OF GENERALIZED STOCHASTIC PROCESSES AND THEIR DERIVATIVES
}

\author{
LEWIS PAKULA
}

ABSTRACT. If $X$ is a generalized stochastic process which is regular in the prediction-theoretic sense then $P(d / d x) X$ is regular for a differential operator $P(d / d x)$. This is used to study sufficient conditions for regularity of stationary processes. On the other hand, an example shows that the derivative of a (nonstationary) deterministic process may be regular.

Let $X$ be a generalized stochastic process with second moments, that is, a continuous linear map $\mathcal{D}\left(\mathbf{R}^{1}\right) \rightarrow H$ where $\mathscr{D}\left(\mathbf{R}^{1}\right)$ is the space of infinitely differentiable functions on $\mathbf{R}^{1}$ with compact support endowed with the usual inductive limit topology and $H$ is a Hilbert space (say of square integrable random variables on a probability space). For $t \in \mathbf{R}$ let $H_{t}=$ closed linear span $\{X(\phi): \operatorname{supp} \phi \subset(-\infty, t)\} ; H_{-\infty}=\bigcap_{t} H_{t} ; H_{\infty}=$ c.l.s. $\bigcup_{t} H_{t}$. If $\langle\cdot ; \cdot\rangle$ denotes the inner product in $H$, then by Schwartz's kemels theorem $B(\phi \otimes \bar{\psi})=\langle X(\phi), X(\psi)\rangle$, where for $\phi, \psi \in \mathcal{D}\left(\mathbf{R}^{1}\right) \phi \otimes \bar{\psi}(x, y)=\phi(x) \bar{\psi}(y)$, defines a distribution $B \in \mathscr{D}^{\prime}\left(\mathbf{R}^{2}\right)$. We say that $X$ is deterministic if $H_{-\infty}=$ $H_{\infty}$ and regular if $H_{-\infty}=\{0\}$. These properties depend only on the covariance distribution $B$ : Let $N=\{\phi: B(\phi \otimes \bar{\phi})=0\}$ and let $H_{B}$ be the Hilbert space obatined by completing the pre-Hilbert space $\mathscr{D}\left(\mathbf{R}^{1}\right) / N$ with the inner product given by $B(\phi \otimes \bar{\psi})$. Let $X_{B}: \mathscr{D}\left(\mathbf{R}^{1}\right) \rightarrow H_{B}$ be the natural map. Then $X$ is regular (resp., deterministic) if and only if $X_{B}$ is regular (resp., deterministic). Moreover, we see that for any distribution $B \in D^{\prime}\left(\mathbf{R}^{2}\right)$ of covariance type, i.e., $B(\phi \otimes \bar{\phi}) \geq 0$ for all $\phi \in \mathscr{D}\left(\mathbf{R}^{1}\right)$, there is g.s.p. $X_{B}$ with $B$ as its covariance distribution. If $\phi_{h}(x)=\phi(h+x)$ and $B\left(\phi_{h} \otimes \psi_{h}\right)=B(\phi \otimes \psi)$ for all $h \in \mathbf{R}^{1}$, we call $X$ stationary. In this case, by the Bochner-Schwartz theorem, there exists a positive Radon measure $\mu$ on $\mathbf{R}^{1}$ (called the spectral measure) and an integer $N \geq 0$ such that

Received by the editors December 7, 1972 and, in revised form, October 17, 1973.

AMS (MOS) subject classifications (1970). Primary 60G20, 60G25.

Key words and phrases. Prediction theory of generalized stochastic processes. 
(1) $B(\phi \otimes \bar{\psi})=\int \hat{\phi}(\hat{\psi})^{-} d \mu, \quad \hat{\phi}(\xi)=\int e^{i x \xi} \phi(x) d x, \int \frac{d \mu(\xi)}{\left(1+\xi^{2}\right)^{N}}<\infty$.

In case (1) holds with $N=0$, that is, $X$ is the g.s.p. associated with an ordinary stationary process with finite spectral measure, it is well known that if $\mu$ is absolutely continuous with respect to Lebesgue measure then $X$ is regular if and only if

$$
\int \frac{\log (d \mu / d \xi)}{1+\xi^{2}} d \xi>-\infty
$$

(See e.g. Deo [2].)

An analogous result for arbitrary $N$ was stated by Rozanov [3] and Balagangadharan [1] but both of their proofs of the sufficiency of (2) contain errors. In this paper a new proof will be given based on

Proposition 1. If $X$ is a regular g.s.p. then $P(d / d x) X$ is regular for any differential operator $P(d / d x)$ with $C^{\infty}$ coefficients.

Proof. Suppose $P(d / d x)=\sum_{i=0}^{n} a_{i}\left(d^{i} / d x^{i}\right)$. Let $P^{*}(d / d x)=$ $\Sigma(-1)^{i} a_{i}\left(d^{i} / d x^{i}\right)$. We define $P(d / d x) X(\phi)=X\left(P^{*}(d / d x) \phi\right)$. Let $H_{t}$ be as above and let $H_{t}^{\prime}=$ c.l:s. $\{P(d / d x) X(\phi)$ : supp $\phi \subset(-\infty, t)\}=$ c.l.s. $\left\{X\left(P^{*}(d / d x) \phi\right)\right.$ : supp $\left.\phi \subset(-\infty, t)\right\}$. But since supp $P^{*}(d / d x) \phi \subset \operatorname{supp} \phi$, it is clear that $H_{t}^{\prime} \subset H_{t}$ and hence $\bigcap_{t}^{\prime} \subset \bigcap_{t}$.

Example. The derivative of a deterministic g.s.p. may be regular. :By Deo [2] a covariance distribution $B$ is deterministic if whenever $B(\phi \otimes \bar{\phi})$ $\geq|U(\phi)|^{2}$ for all $\phi \in \mathscr{D}\left(\mathbf{R}^{1}\right)$ where $U \in \mathscr{D}^{\prime}\left(\mathbf{R}^{1}\right)$ vanishes on a left half-line, then $U=0$. Let $X$ be a g.s.p. with covariance $V \otimes \bar{V}$ where $V=1+F$ and $F$ is the Heaviside function. $|V(\phi)|^{2} \geq|U(\phi)|^{2}$ for all $\phi \in \mathscr{D}\left(\mathbf{R}^{1}\right)$ and $U \neq 0$ implies $V=c U$ for some $c \neq 0$. Hence $X$ is deterministic. Its derivative has covariance distribution $\delta_{0} \otimes \delta_{0}$ and so is regular. The derivative of a stationary deterministic g.s.p. is deterministic however, since it can be shown, using Proposition 2 below, that a stationary $X$ is deterministic if and only if

$$
\int \frac{\log \left(d \mu_{a} / d \xi\right)}{1+\xi^{2}} d \xi=-\infty
$$

where $\mu_{a}$ is the absolutely continuous part of $\mu$ (see Rozanov [3]). But the spectral measure of $X^{\prime}$ is $\xi^{2} \mu$ so the result follows from the fact that $\int\left(\left(\log \xi^{2}\right) /\left(1+\xi^{2}\right)\right) d \xi$ is finite.

Proposition 2. If $X$ is a stationary g.s.p. with absolutely continuous 
spectral measure $\mu$ and (2) holds, then $X$ is regular.

Proof. Since (1) holds for some $N$ there is a positive $g \in L^{1}\left(\mathbf{R}^{1}\right)$ such that $d \mu / d \xi=\left(1+\xi^{2}\right)^{N} g(\xi)=(1-i \xi)^{N}(1+i \xi)^{N} g(\xi)$. Denote the inverse Fourier transform acting on tempered distributions by $\mathcal{F}^{-1}$. Then

$$
\mathfrak{F}^{-1} \mu=(1-(d / d x))^{N}(1+(d / d x))^{N \mathcal{F}-1} g
$$

and $B(\phi \otimes \bar{\psi})=\mathcal{F}^{-1} \mu(\phi * \tilde{\psi})$ is the covariance distribution of $X$ where $*$ denotes convolution and $\tilde{\psi}(x)=\overline{\psi(-x)}$. Let $B^{\prime}(\phi \otimes \bar{\psi})=\mathfrak{F}^{-1} g(\phi * \widetilde{\psi})$. Since $B^{\prime}(\phi \otimes \bar{\phi}) \geq 0, B^{\prime}$ is the covariance distribution of some stationary g.s.p. $Y$ with finite spectral measure $g(\xi) d \xi$. Then $B$ is the covariance of $(1+(d / d x))^{N} Y$. Now if $Y$ were regular, by Proposition $1, X$ would be also since this property depends only on the covariance. But

$$
\int \frac{\log (g) d \xi}{1+\xi^{2}}=\int \frac{\log (d \mu / d \xi)}{1+\xi^{2}} d \xi-\int \frac{\log \left(1+\xi^{2}\right)^{N}}{1+\xi^{2}} d \xi>-\infty .
$$

Hence $Y$ is regular.

The author thanks the referee for his suggestions.

\section{REFERENCES}

1. K. Balagangadharan, The prediction theory of stationary random distributions, Mem. Coll. Sci. Univ. Kyoto Ser. A Math. 33 (1960/61), 243-256. MR 23 \#A682.

2. C. M. Deo, Prediction theory of non-stationary processes, Sankyhā Ser. A 27 (1965), 113- 132. MR 36 \#941.

3. Ju. Rozanov, On the extrapolation of generalized stationary random processes, Teor. Verojatnost. i Primenen. 4 (1959), 465-471 = Theor. Probability Appl. 4 (1959), 426-431. MR $22 \# 6018$.

DEP ARTMENT OF MATHEMATICS, UNIVERSITY OF RHODE ISLAND, KINGSTON, RHODE ISLAND 02881 